\title{
Thymoma by Masaoka-Koga Stage
}

National Cancer Institute

\section{Source}

National Cancer Institute. Thymoma by Masaoka-Koga Stage. NCI Thesaurus. Code

C112006.

A staging system for thymoma based on the anatomic extent of disease at the time of surgery. 